\title{
LA CONTRATACIÓN COMPLEMENTARIA: MEDIDA EXCEPCIONAL PARA LA CONTINUIDAD DE LAS PRESTACIONES A TRAVÉS DE UNA NUEVA CONTRATACIÓN
}

\author{
Roy Álvarez ChuquilLanquI \\ Organismo Supervisor de las Contrataciones del Estado, Lima, Perú
}

Recibido: 2/1/2020 - Aprobado: 9/1/2020

doi: https://doi.org/10.26439/iusetpraxis2020.n50-51.5051

RESUMEN. En el presente trabajo, el autor aborda la figura de la contratación complementaria prevista en la normativa de contrataciones del Estado, y realiza un análisis de este mecanismo excepcional en virtud de los criterios vertidos por el Organismo Supervisor de las Contrataciones del Estado (OSCE).

PALABRAS CLAVE: OSCE / contrataciones del Estado / contrato / prestaciones

\section{CONTRACT RENEWAL: AN EXCEPTIONAL MEASURE FOR THE CONTINUITY OF PROVISION OF SERVICES THROUGH A NEW CONTRACT}

ABSTRACT. In this paper, the author addresses the concept of contract renewal provided for in government procurement regulations, and analyzes this exceptional mechanism based on the criteria established by the Government Procurement Supervising Agency (OSCE).

KEYWORDS: OSCE / government procurement / contract / provision of services 


\title{
IDEAS INICIALES. INTRODUCCIÓN
}

El quehacer de la administración pública trae consigo un importante componente contractual, ya que a través de este medio pueden adquirirse los bienes, servicios y obras requeridos por las entidades públicas (en adelante, las entidades) para el cumplimiento de sus funciones.

A diferencia de la contratación tradicional, la contratación pública posee un tratamiento singular en función de la necesidad pública que busca atender. Al respecto, el Tribunal Constitucional ha señalado que

\begin{abstract}
la contratación estatal tiene un cariz singular que lo diferencia de cualquier acuerdo de voluntades entre particulares, ya que, al estar comprometidos recursos y finalidades públicas, resulta necesaria una especial regulación que permita una adecuada transparencia en las operaciones. (Expediente 020-2003-Al/TC, 2004, numeral 11)
\end{abstract}

Dicha especial regulación se encuentra recogida en la normativa de contrataciones del Estado', la cual establece -entre otros aspectos - los requisitos y procedimientos que deben emplearse tanto para la selección y contratación de un determinado proveedor como para la atención de la necesidad prevista por la entidad durante la ejecución contractual.

Sobre este punto ha de indicarse que la suscripción de un contrato entre una entidad y un proveedor del Estado - el cual en ese momento adquiere la denominación de contratista-constituye el hito que pone fin a la fase selectiva y permite el punto de inicio de la fase de ejecución contractual, en la cual las partes desarrollan sus respectivas prestaciones a satisfacción de su contraparte, con lo cual alcanzan la finalidad de la contratación.

No obstante, culminada la ejecución de las prestaciones, puede darse el caso de que - de manera excepcional ${ }^{2}$ - una entidad requiera la continuidad de dichas prestaciones para la atención de una nueva necesidad que por sus características no amerite un procedimiento de selección o, de corresponder, dicha contratación se desarrolle mientras se elija al nuevo contratista.

Así, por ejemplo, una entidad convoca un procedimiento de selección para la contratación del servicio de seguridad y vigilancia de sus sedes a nivel nacional por el periodo de un año. Producto de ello, un determinado proveedor obtiene la buena pro y suscribe

1 De conformidad con lo indicado por el OSCE en constantes opiniones, la normativa de contrataciones del Estado se encuentra conformada por la Ley de Contrataciones del Estado, su reglamento y las demás normas de carácter reglamentario emitidas por el OSCE.

2 Sobre la naturaleza excepcional de esta figura, pueden revisarse las Opiniones 102-2018/DTN, 040-2017/DTN y 031-2016/DTN. 
contrato. El contratista desarrolla con normalidad su prestación y, estando cerca de la culminación del plazo de ejecución contractual, la entidad advierte que el procedimiento de selección convocado para elegir al nuevo proveedor se ha retrasado y que a la fecha de culminación del plazo de ejecución de la contratación original no se contaría con el nuevo proveedor adjudicado, con lo cual se genera una afectación al aprovisionamiento oportuno del referido servicio.

Ante dicha eventualidad, la normativa de contrataciones del Estado contempla la figura de la contratación complementaria como un mecanismo excepcional aplicable en la contratación de bienes y servicios en general, que permite contratar de manera directa al mismo contratista para que desarrolle la misma prestación manteniendo las condiciones que formaron parte de la contratación original.

\section{ASPECTOS GENERALES DEL CONTRATO COMPLEMENTARIO}

\section{Contratación inmediata sin fase de selección}

Dentro del proceso de contratación existen fases que permiten a la entidad desarrollar la contratación de su necesidad. Así, de manera previa se planifica y formula la voluntad estatal para luego, mediante un método de contratación, elegir al proveedor con el cual se suscribirá el contrato, con el fin de reducir la discrecionalidad en la selección del proveedor.

No obstante, la posibilidad de contratar complementariamente constituye un supuesto de excepción a la obligación de las entidades de convocar procedimientos de selección para contratar a los proveedores que les brindarán los bienes o servicios en general necesarios para el cumplimiento de sus funciones. Así, Morón (2016) señala que

la normativa privilegia el principio de eficiencia por sobre el principio de libre concurrencia en el entendido que el proveedor ya fue elegido sobre bases objetivas en un proceso anterior y que resulta conveniente para la obtención pronta de los medios que necesita la Administración (bienes y servicios para el cumplimiento de sus fines), seleccionar ya a quien ha evidenciado que sus bienes o servicios satisfacen suficientemente los requisitos adecuados de precio, calidad, plazo de entrega y uso final. (p. 682)

En ese sentido, la contratación complementaria, al ser una contratación sin procedimiento de selección, debe aplicarse de forma restrictiva y únicamente cuando se cumplan las condiciones establecidas en la normativa de contrataciones del Estado. 


\section{Libre consentimiento de las partes}

La contratación complementaria constituye un nuevo contrato, pero con el mismo contratista inicial y en las mismas condiciones que el contrato original. Por lo tanto, para la suscripción de este nuevo contrato es necesario contar, en principio, con la voluntad de la entidad de continuar con dichas prestaciones y la voluntad del contratista de brindarlas bajo las mismas condiciones, el cual no se encuentra en la obligación de suscribirlo ${ }^{3}$.

Asimismo, debe indicarse que la contratación complementaria se materializa con la voluntad de las partes plasmada en un nuevo contrato y no requiere de un documento de aprobación o autorización correspondiente ${ }^{4}$, como sí es aplicable en otras figuras de la ejecución contractual ${ }^{5}$.

\section{Justificación de la contratación complementaria}

Tal como se indicó en el acápite anterior, la decisión de suscribir un contrato complementario nace de la propia voluntad de las partes plasmada en el nuevo contrato sin requerirse ningún documento autoritativo adicional.

Ahora bien, el hecho de que no se necesite un documento que lo autorice no significa que la contratación complementaria pueda carecer de la justificación debida. Así, conforme a lo indicado por el OSCE en el criterio previsto en la Opinión 085-2019/DTN, el área usuaria ${ }^{6}$ de la contratación debe brindar el sustento necesario que permita requerir y justificar la finalidad pública de una contratación complementaria.

Por su parte, un aspecto relevante recogido en la mencionada Opinión 085-2019/ DTN es la posibilidad de que el área requirente de la contratación complementaria sea una distinta al área usuaria de la contratación original, en el entendido de que

la normativa de contrataciones del Estado no ha previsto, como requisito para el empleo de la contratación complementaria, que esta deba ser requerida y/o sustentada por la misma área usuaria que otorgó la conformidad del bien o servicio en general que fue contratado originalmente. (numeral 3.4)

En tal sentido, la contratación complementaria puede sustentarse por un área usuaria distinta a la prevista en la contratación inicial, siempre que sea requerida por un

3 Al respecto, puede consultarse la Opinión 056-2014/DTN, la cual concluye que "la contratación complementaria, al determinar la suscripción de un nuevo contrato con el mismo contratista y en las mismas condiciones del contrato original, requiere contar con el consentimiento previo de las partes; por lo que, el contratista no se encuentra obligado a suscribirlo, debiendo más bien producirse un acuerdo o consentimiento de este con la Entidad".

4 Véase este aspecto en la Opinión 010-2016/GTN.

5 Tales como la aprobación de adicionales, reducciones o contrataciones directas.

6 Es decir, aquella cuyas necesidades pretenden ser atendidas con dicha contratación, o la que canaliza los requerimientos formulados por otras dependencias. 
área dentro de la entidad, se justifique la finalidad pública de tal contratación y esta se oriente al cumplimiento de las funciones de la entidad.

\section{Prestaciones divisibles}

Si bien la contratación complementaria puede aplicarse en los objetos contractuales de bienes y servicios en general, no en todas estas prestaciones resultará procedente dicha figura. Así, por ejemplo, si una entidad adquiere un equipo médico de gran complejidad cuyo monto corresponde a un procedimiento de adjudicación simplificada, no procedería en este caso aplicar una contratación complementaria (contratación menor), ya que dicha contratación original no tiene la calidad de ser divisible en una prestación inferior a la total contratada.

Así, solo serán procedentes las contrataciones complementarias cuando, dentro de los requisitos previstos en la normativa de contrataciones del Estado, se realicen respecto a bienes o servicios en general que tengan la calidad de ser divisibles, y así suscribir un nuevo contrato menor que integra elementos desarrollados en la contratación original.

\section{Necesidad sobreviniente}

Se ha mencionado que la contratación complementaria tiene su origen en un contrato anterior suscrito entre una entidad y un proveedor válidamente seleccionado. Al respecto, el hecho de que esta figura se denomine contratación complementaria no significa que los bienes o servicios en general a contratarse tengan una vinculación de complementariedad con los bienes o servicios en general anteriormente prestados, sino que mediante ella se busca atender una nueva necesidad de la entidad, cuya contratación complementa a la anterior.

En ese sentido, la contratación complementaria nace como una nueva necesidad sobreviniente a la ya ejecutada, que, si bien se vincula con la anterior al compartir determinados elementos, representa una nueva contratación.

\section{DEFINICIÓN DEL CONTRATO COMPLEMENTARIO}

El contrato complementario constituye —según sostiene Morón (2016)—

la aptitud o habilitación legal para que las entidades puedan concertar de manera directa contrataciones de aprovisionamiento de bienes o servicios - sin acudir al procedimiento que por el monto y materia correspondería- con un proveedor anterior siempre que se trate del mismo objeto contractual y bajo las mismas condiciones anteriores. (p. 682) 
Por su parte, Martínez (2008) señala lo siguiente:

Este contrato complementario es un reflejo directo del contrato inicial que lo informa, no pudiendo introducirse nuevos elementos de negociación o variantes, puesto que el único elemento discrecional que conservan las partes, en adición a la propia voluntad de obligarse a dicho contrato, es el de establecer su duración y volumen, cuyo límite es el $30 \%$ del contrato original. (p. 128)

En atención a estas definiciones, podemos señalar que el contrato complementario es una herramienta excepcional que permite a la entidad realizar una nueva contratación - de manera directa y sin desarrollar el procedimiento de selección correspondientecon el proveedor contratado inicialmente, y se contemplan en esta nueva contratación las condiciones de la original conforme a los límites y restricciones establecidos en la normativa de contrataciones del Estado.

Así, cuando la entidad, luego de culminada la ejecución de un contrato, mantiene la misma necesidad - la cual resulta ser inmediata y sobreviniente a la anterior-, puede emplear esta figura para contar con la prestación en el tiempo que tarde el desarrollo del procedimiento de selección correspondiente para la nueva contratación, es decir, emplear la contratación complementaria como una medida temporal hasta contar con el contrato derivado del nuevo procedimiento de selección convocado.

\section{Nuevo contrato}

Plazo de ejecución

contractual

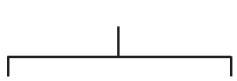

Contratación complementaria

Contratación original
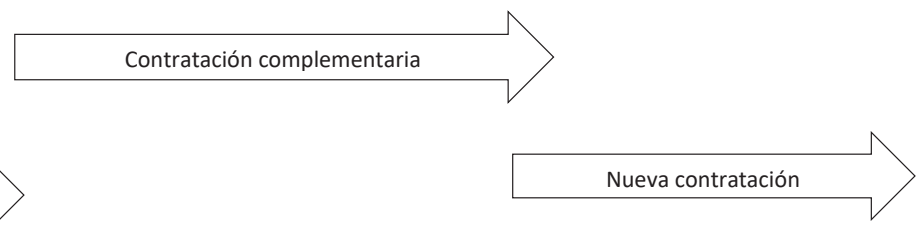

Derivado del

procedimiento de

selección convocado

\section{Elaboración propia}

Por el contrario, cuando la contratación complementaria permite cubrir la necesidad de manera total y no resulta necesario convocar un nuevo procedimiento de selección, como sucede en la contratación de bienes o servicios no reiterativos o permanentes, el contrato complementario permitirá atender la necesidad en conjunto. 


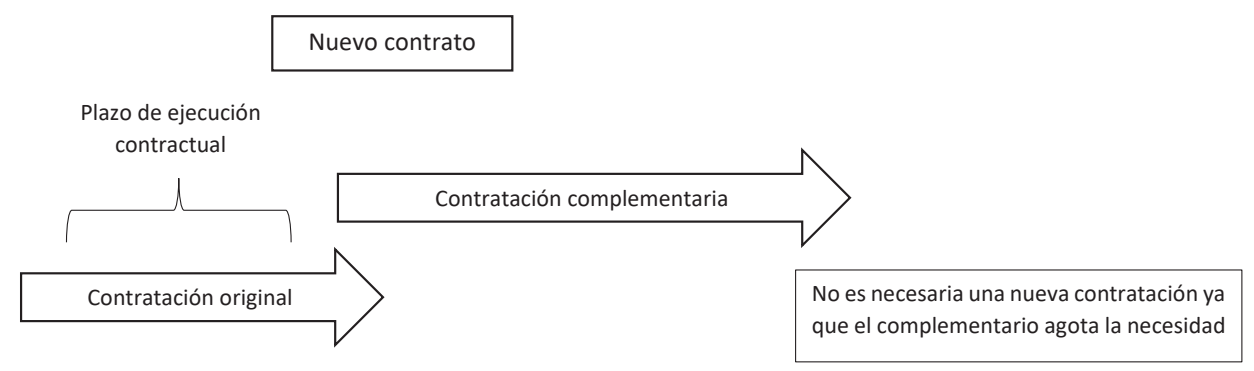

Elaboración propia

\section{NORMATIVA APLICABLE AL CONTRATO COMPLEMENTARIO}

Considerando que el contrato complementario constituye una nueva contratación, surge la duda sobre la normativa que debe aplicarse cuando se haya producido una variación respecto a la normativa vigente en el contrato original.

Al respecto, considerando la regulación constitucional sobre la aplicación de las normas en el tiempo ${ }^{7}$, nuestro ordenamiento jurídico se rige por la teoría de los hechos cumplidos, por la cual la ley es obligatoria desde su entrada en vigencia, esto es, desde el día siguiente de su publicación en el diario oficial, y se aplica a las consecuencias de las relaciones y situaciones jurídicas existentes, salvo disposición expresa de la misma ley que posterga su vigencia en todo o en parte, o que permite que la legislación precedente siga produciendo efectos de manera ultractiva.

Sobre el particular, el numeral 11 de la Sentencia del Tribunal Constitucional derivada del Expediente 0002-2006-PI/TC establece que

Díez-Picazo [...] sostiene que "en el momento en que una ley entra en vigor, despliega, por definición, sus efectos normativos y debe ser aplicada a toda situación subsumible en su supuesto de hecho; luego no hay razón alguna por la que deba aplicarse la antigua ley a las situaciones, aún no extinguidas, nacidas con anterioridad".

7 El artículo 103 de la Constitución Política del Perú establece que "la ley, desde su entrada en vigencia, se aplica a las consecuencias de las relaciones y situaciones jurídicas existentes y no tiene fuerza ni efectos retroactivos; salvo, en ambos supuestos, en materia penal cuando favorece al reo. La ley se deroga solo por otra ley". Por su parte, el artículo 109 de la carta magna dispone lo siguiente: "La ley es obligatoria desde el día siguiente de su publicación en el diario oficial, salvo disposición contraria de la misma ley que posterga su vigencia en todo o en parte". 
En tal sentido, en vista de que la contratación complementaria constituye un nuevo contrato, en este se aplica la normativa vigente al momento de su suscripción. Similar criterio mantiene el OSCE en las Opiniones 040-2017/DTN, 088-2019/DTN, entre otras.

\section{REQUISITOS DEL CONTRATO COMPLEMENTARIO}

El artículo 174 del Reglamento de la Ley de Contrataciones del Estado, aprobado mediante el Decreto Supremo 344-2018-EF, establece la regulación normativa de la contratación complementaria y precisa los requisitos necesarios para que esta sea procedente. Así, el referido artículo fija las siguientes condiciones para el empleo de la contratación complementaria: i) que esta se realice dentro de los tres meses posteriores a la culminación del plazo de ejecución del contrato; ii) con el mismo contratista; iii) por un monto no mayor al $30 \%$ del monto del contrato original; iv) por única vez; v) en tanto culmine el procedimiento de selección convocado; vi) que se trate del mismo bien o servicio en general antes contratado; vii) que el contratista preserve las condiciones que dieron lugar a la adquisición o contratación, y viii) que su celebración no sea respecto a contratos de ejecución de obras ni de consultorías (incluidas las de obra), y tampoco de contrataciones directas.

\section{Contratación complementaria}
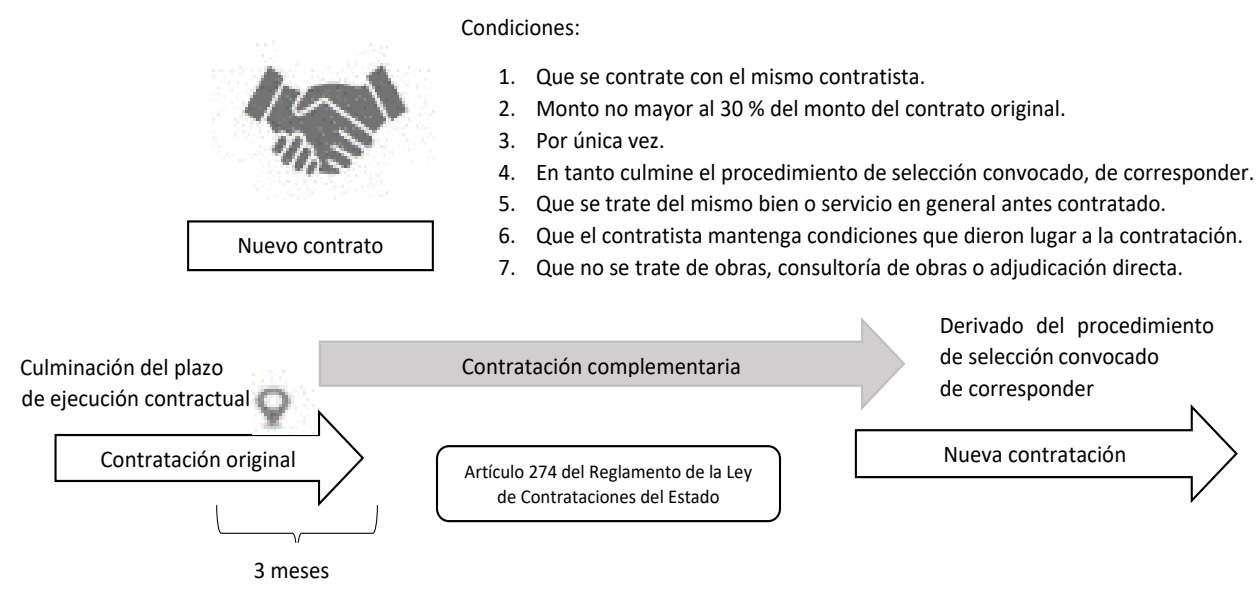

Elaboración propia

- Que se realice dentro de los tres meses posteriores a la culminación del plazo de ejecución del contrato

Una de las condiciones para desarrollar una contratación complementaria es que esta sea suscrita dentro de los tres meses posteriores a la culminación 
del plazo de ejecución contractual de la contratación original. El hecho de que se cuente con estos tres meses no significa que la prestación del contrato complementario deba efectuarse en dicho periodo, sino que en este las partes se encuentran habilitadas para perfeccionar el contrato complementario.

Para conocer el punto de partida de donde se contabilizan los referidos tres meses, es preciso mencionar que el plazo de ejecución contractual es el periodo en que el contratista se ha obligado a ejecutar las prestaciones a su cargo. No debe confundirse el referido plazo con el de vigencia del contrato, el cual está referido al periodo de existencia o vigor de las obligaciones del contrato ${ }^{8}$.

En ese sentido, culminado el plazo de ejecución contractual -lo que no incluye el plazo correspondiente a la entidad para otorgar la conformidad y efectuar el pago-, las partes del contrato original cuentan con hasta tres meses para suscribir el contrato complementario.

- Que se perfeccione con el mismo contratista

Si bien la contratación complementaria permite a la entidad contratar de manera directa a un proveedor para ejecutar prestaciones ya desarrolladas, esta nueva contratación debe efectuarse necesariamente con el mismo contratista, siempre que este manifieste su voluntad de suscribir el nuevo contrato.

Ahora bien, considerando que la contratación complementaria constituye una nueva relación contractual que se materializa a través de la celebración de un nuevo contrato, si al momento de suscribirlo el contratista se encuentra impedido para contratar con el Estado, tal situación imposibilitaría la suscripción del contrato complementario.

- Que la contratación se desarrolle por un monto no mayor al $30 \%$ del monto del contrato original

Uno de los requisitos para la suscripción de un contrato complementario es que el monto de este no supere el $30 \%$ del monto del contrato original. Al respecto, el Anexo de Definiciones del Reglamento de la Ley de Contrataciones del Estado señala que el "contrato original" es aquel suscrito como consecuencia del otorgamiento de la buena pro en las condiciones establecidas en las bases y la oferta ganadora?.

8 Es necesario precisar que la vigencia del contrato no finaliza con la ejecución de las prestaciones por parte del contratista, pues una vez vencido dicho plazo aún deben llevarse a cabo algunos actos adicionales, como la conformidad y el pago.

9 A diferencia del contrato actualizado o vigente, el cual es definido como el contrato original afectado por las variaciones realizadas por los reajustes, las prestaciones adicionales, la reducción de prestaciones o por ampliación o reducción del plazo, u otras modificaciones del contrato. 
En tal sentido, el monto de un contrato complementario tiene como límite cuantitativo el $30 \%$ del monto del contrato suscrito según las condiciones señaladas en las bases y en la oferta ganadora (contrato original), sin considerar para tal efecto las variaciones de precio que pudieran haberse producido, tales como las generadas por prestaciones adicionales, reducción de prestaciones, reajustes, entre otras modificaciones efectuadas durante su ejecución.

- Que la contratación se realice por única vez

Considerando el carácter excepcional de esta figura, solo puede desarrollarse en un único momento. Así, incluso cuando no se haya empleado el límite porcentual máximo para desarrollar una contratación complementaria, no podrán llevarse a cabo posteriormente nuevas contrataciones de este tipo, ya que la normativa ha considerado que se desarrolle en una sola oportunidad.

En tal sentido, corresponde a la entidad identificar las prestaciones requeridas y desarrollar la contratación complementaria en una sola ocasión, para garantizar el cumplimiento de las funciones, los servicios, las actividades o las operaciones que la entidad tiene a su cargo, y contratar únicamente la parte necesaria para cubrir un eventual desabastecimiento.

- Que la contratación se realice en tanto culmine el procedimiento de selección convocado

En la medida en que la contratación complementaria permite que la entidad satisfaga la necesidad de abastecerse de determinados bienes o servicios en general entre una contratación culminada y otra en curso, la entidad deberá contratar únicamente la parte necesaria para cubrir un eventual desabastecimiento y en tanto culmine el procedimiento de selección ya convocado. Así, dicho requisito supone la existencia de un procedimiento de selección convocado de forma previa a la suscripción del contrato complementario, con la finalidad de garantizar el desarrollo de las actividades a cargo de la entidad, en tanto se seleccione al proveedor que cubrirá dicho requerimiento.

Ahora bien, la normativa es clara al indicar la necesidad de contar con la convocatoria del procedimiento de selección de forma previa a la suscripción del contrato complementario, por lo que este no podría suscribirse si el proceso de contratación se encontrase en la fase de planificación y actuaciones preparatorias.

No obstante, tal requisito no siempre será exigido, ya que, de conformidad con el numeral 174.2 del artículo 174 del Reglamento de la Ley de Contrataciones del Estado, no será necesario llevar a cabo la nueva convocatoria cuando con dicha contratación complementaria se agote la necesidad de la entidad, lo cual debe ser sustentado por el área usuaria al formular su requerimiento. 
- Que la contratación se realice respecto al mismo bien o servicio en general antes contratado

Para que la contratación complementaria sea procedente, la prestación que es objeto de contratación debe corresponder al bien o servicio en general que fue materia de la contratación original. En ese sentido, independientemente de si se trata de bienes o servicios, la contratación complementaria ha de comprender las mismas prestaciones contratadas (bien o servicio en general), sin existir la posibilidad de que al momento del perfeccionamiento se puedan incorporar nuevas prestaciones.

- Que el contratista preserve las condiciones que dieron lugar a la adquisición o contratación

Si bien la contratación complementaria determina la celebración de un nuevo contrato, aunque con el mismo contratista y sobre los mismos bienes o servicios en general, resulta necesario que el contrato complementario incorpore todas las condiciones que dieron lugar a la contratación original y aquellas en las que esta culminó su ejecución.

Ahora bien, es preciso mencionar que durante la ejecución contractual el contrato puede sufrir modificaciones expresamente permitidas en la normativa de contrataciones del Estado, tales como adicionales, reducción u otras modificaciones contractuales. Así, tal como lo establece la Opinión 152-2015/DTN, la verificación de la identidad del objeto y de las condiciones del contrato complementario debe realizarse respecto a las prestaciones y condiciones vigentes al momento en que culminó su ejecución.

En tal sentido, las condiciones establecidas en el contrato original - lo que incluye las modificaciones a él- se mantienen y, por lo tanto, son de aplicación al contrato complementario que se suscriba, con excepción del monto del contrato original, el cual no puede ser mayor al treinta $30 \%$ de este.

- Que su celebración no sea respecto a contratos de ejecución de obras ni de consultorías, y tampoco de contrataciones directas

El legislador ha optado porque la contratación complementaria se emplee en la contratación de bienes y servicios en general en la medida en que estos objetos contractuales pueden ajustarse a las condiciones descritas en el artículo 174 del Reglamento de la Ley de Contrataciones del Estado.

En efecto, la contratación complementaria no es posible para el caso de obras, ya que por la especialidad no resultaría razonable contratar las mismas prestaciones para la construcción de un nuevo hospital o quizá una nueva obra de saneamiento, teniendo en cuenta que cada obra, por muy similar que sea a otra 
previamente desarrollada, mantiene características independientes y debe contar con su propio expediente técnico de obra. Por su parte, se observa que en la contratación de las consultorías - que incluye consultoría en general y consultoría de obras- no es procedente la figura de la contratación complementaria dado que no podría desarrollarse una contratación menor a la inicialmente efectuada, en la medida en que una consultoría no posee la calidad de ser divisible y constituye un único todo.

En ese sentido, la contratación complementaria puede desarrollarse para la contratación de bienes o servicios en general, pero no en todos estos objetos, puesto que es necesario que dicha prestación tenga la calidad de ser divisible, entre otras condiciones establecidas en la normativa de contrataciones del Estado.

Por su parte, considerando que la contratación complementaria es un procedimiento excepcional que permite atender necesidades de orden coyuntural, el contratista debería ser elegido mediante un procedimiento competitivo que dé origen a la contratación inicial. En tal sentido, la figura de la contratación complementaria no puede ser empleada en los contratos derivados de una contratación directa ${ }^{10}$, en la medida en que en este tipo de contrataciones existe una privación del principio de libre competencia.

\section{DIFERENCIA ENTRE EL CONTRATO COMPLEMENTARIO Y LAS PRESTACIONES ADICIONALES}

En la ejecución contractual es posible aplicar distintas figuras que permiten a la entidad alcanzar la finalidad del contrato; sin embargo, con el fin de extender la provisión de las prestaciones contratadas, se observa que las entidades emplean equivocadamente la figura de la contratación de prestaciones adicionales y la contratación complementaria.

En principio, debe indicarse que la contratación de prestaciones adicionales es una prerrogativa con la que cuenta la entidad a fin de ordenar al contratista efectuar prestaciones adicionales a las originalmente pactadas hasta por el límite establecido en la normativa de contrataciones del Estado. Dichas prestaciones tienen como objetivo alcanzar la finalidad del contrato, lo cual implica perseguir el propósito de satisfacer la necesidad pública que originó dicha contratación, y el costo de dichas prestaciones adicionales, en los contratos de bienes o servicios, se determina sobre la base de las especificaciones técnicas o términos de referencia, según corresponda, y de las condiciones y precios pactados en el contrato, 0 -en su defecto- por acuerdo entre las partes.

10 La contratación directa es un procedimiento de selección de carácter no competitivo, puesto que, por razones coyunturales, económicas o de mercado, la entidad pública requiere contratar directamente con un determinado proveedor para satisfacer su necesidad. 
Por su parte, a diferencia de lo anterior, la contratación complementaria constituye una nueva contratación -en el entendido de que la original alcanzó la finalidad debidaque tiene su origen en una circunstancia extraordinaria externa sobreviniente al contrato ya ejecutado, que permite realizarla dentro de los tres meses posteriores a la culminación del plazo de ejecución contractual.

Por otro lado, a diferencia de la prestación adicional, la contratación complementaria no resulta ser obligatoria, por lo que requiere la concurrencia de voluntades y la aceptación del anterior proveedor.

Tabla 1

Diferencia entre prestaciones adicionales y contratación complementaria

\begin{tabular}{|c|c|}
\hline Prestaciones adicionales & Contratación complementaria \\
\hline Se aplican a un contrato en ejecución. & Representa un nuevo contrato. \\
\hline $\begin{array}{l}\text { Buscan alcanzar la finalidad de la contratación } \\
\text { originaria. }\end{array}$ & $\begin{array}{l}\text { Corresponde a una necesidad sobreviniente a la } \\
\text { contratación originaria. }\end{array}$ \\
\hline Se ejecutan de manera obligatoria. & Requiere el consentimiento de las partes. \\
\hline $\begin{array}{l}\text { Se aplican para todos los objetos } \\
\text { contractuales. }\end{array}$ & Se aplica solo para bienes y servicios en general. \\
\hline $\begin{array}{l}\text { Se aprueba como máximo hasta el } 25 \% \text { del } \\
\text { monto del contrato original en el caso de } \\
\text { bienes y servicios. }\end{array}$ & $\begin{array}{l}\text { Se aprueba como máximo hasta el } 30 \% \text { del monto del } \\
\text { contrato original. }\end{array}$ \\
\hline $\begin{array}{l}\text { Pueden ser aprobadas más de una vez en la } \\
\text { medida en que no excedan el monto máximo. }\end{array}$ & Se realiza solo una vez. \\
\hline
\end{tabular}

Elaboración propia

En ese sentido, las figuras de las prestaciones adicionales y las contrataciones complementarias muestran marcadas diferencias, en principio, por el ámbito donde se aplican, ya sea a la contratación original (prestaciones adicionales) o a la nueva contratación (contratación complementaria). Asimismo, es preciso mencionar que no es un requisito previo haber optado por la ejecución de prestaciones adicionales para luego emplear la contratación complementaria, ya que cada una de estas figuras se aplica cuando se configuren los supuestos previstos en la normativa de contrataciones del Estado.

\section{APLICACIÓN DE LAS FIGURAS DE LA EJECUCIÓN CONTRACTUAL}

En la medida en que la contratación complementaria determina la suscripción de un nuevo contrato, resulta posible que en dicha contratación se apliquen las figuras propias de la ejecución contractual previstas en la normativa de contrataciones del Estado. Tal criterio ha sido establecido por el OSCE en diversas opiniones, como por ejemplo en la 
Opinión 102-2018/DTN, en la que se permite la aplicación de la figura de ampliación de plazo en una contratación complementaria.

En ese sentido, las figuras aplicables durante la ejecución contractual pueden ser empleadas en la ejecución del contrato complementario siempre que se cumpla con cada uno de los requisitos establecidos en la normativa de contrataciones del Estado.

Considerando lo anterior, en una contratación complementaria es posible la aplicación de prestaciones adicionales; sin embargo, bajo un reciente criterio recogido en la Opinión 228-2019/DTN se ha precisado lo siguiente:

\footnotetext{
En el supuesto en que una entidad hubiese determinado la necesidad de aprobar prestaciones adicionales durante la ejecución de un contrato complementario, deberá cuidar -entre otros aspectos- que: i) los adicionales sean necesarios para alcanzar la finalidad del contrato y no excedan el $25 \%$ del monto de la contratación complementaria; y ii) el monto del contrato complementario -incluido los adicionales- no exceda el $30 \%$ del monto del contrato original, conforme a lo establecido en el artículo 150 del Reglamento.
}

Sobre el primer elemento no se presenta mayor complejidad, ya que, en efecto, para que proceda la ejecución de prestaciones adicionales en el caso de bienes o servicios tales no pueden exceder el $25 \%$ del monto del contrato original, que para el presente caso representa el monto de la contratación complementaria.

No obstante, el segundo elemento sí posee mayor relevancia, ya que está fijando como tope máximo del contrato complementario, incluidas las prestaciones adicionales, el $30 \%$ del monto del contrato original. Así, en caso de que se haya optado por suscribir el contrato complementario por el tope máximo correspondiente $(30 \%$ del monto del contrato original), no resultaría procedente la aprobación de prestación adicional alguna.

\section{CONCLUSIONES}

La contratación complementaria constituye una nueva relación contractual entre el contratista y la entidad, que se materializa a través de la celebración de un nuevo contrato y que es distinto e independiente de aquel que las partes celebraron originalmente. Así, dicha figura permite proveer a la entidad los mismos bienes y servicios en general que esta requiere para atender una determinada necesidad, hasta que culmine - de ser el caso- el procedimiento de selección convocado para tal efecto.

Pese a que las contrataciones complementarias y la ejecución de prestaciones adicionales permiten satisfacer las necesidades de bienes y servicios que pueda tener la entidad, la contratación complementaria encuentra su justificación en la necesidad de atender una situación extraordinaria y externa a la ejecución de un contrato ya culminado. 
De conformidad con el reciente criterio del OSCE recogido en la Opinión 228-2019/ DTN, el límite porcentual aplicado para la contratación complementaria, correspondiente hasta el $30 \%$ del monto del contrato original, representa el límite máximo no solo al momento de perfeccionar el contrato complementario, sino también durante su ejecución. Por lo tanto, de aprobarse prestaciones adicionales al contrato complementario, el monto de este - incluidos los adicionales- no puede exceder el $30 \%$ del monto del contrato original.

\section{REFERENCIAS}

Expediente 0002-2006-PI-TC (Lima). (2007). Tribunal Constitucional: Pleno Jurisdiccional. Recuperado de http://www.tc.gob.pe/jurisprudencia/2007/00002-2006-Al.pdf

Expediente 020-2003-AI/TC (Lima). (2004). Tribunal Constitucional: Pleno Jurisdiccional. Recuperado de https://www.tc.gob.pe/jurisprudencia/2004/00020-2003-Al.html

Martínez, M. (mayo del 2008). El contrato complementario en el régimen de contratación pública. Revista Jurídica del Perú. Normas Legales, (87), 125-133.

Morón, J. (2016). La contratación estatal. Lima: Gaceta Jurídica.

Opinión 010-2016/DTN. (2016). Organismo Supervisor de las Contrataciones del Estado: Dirección Técnico Normativa. Recuperado de https://www.gob.pe/institucion/ osce/informes-publicaciones/737629-opinion-n-010-2016-dtn

Opinión 056-2014/DTN. (2014). Organismo Supervisor de las Contrataciones del Estado: Dirección Técnico Normativa. Recuperado de https://www.gob.pe/institucion/ osce/informes-publicaciones/738082-opinion-n-056-2014-dtn

Opinión 085-2019/DTN. (2019). Organismo Supervisor de las Contrataciones del Estado: Dirección Técnico Normativa. Recuperado de https://www.gob.pe/institucion/ osce/informes-publicaciones/290921-opinion-n-085-2019-dtn

Opinión 102-2018/DTN. (2018). Organismo Supervisor de las Contrataciones del Estado: Dirección Técnico Normativa. Recuperado de https://www.gob.pe/institucion/ osce/informes-publicaciones/737235-opinion-n-102-2018-dtn

Opinión 152-2015/DTN. (2015). Organismo Supervisor de las Contrataciones del Estado: Dirección Técnico Normativa. Recuperado de https://www.gob.pe/institucion/ osce/informes-publicaciones/737981-opinion-n-152-2015-dtn

Opinión 228-2019/DTN. (2019). Organismo Supervisor de las Contrataciones del Estado: Dirección Técnico Normativa. Recuperado de https:/www.gob.pe/institucion/ osce/informes-publicaciones/429467-opinion-n-228-2019-dtn 